\title{
Melatonin reverses the enhanced oxidative damage to membrane lipids and improves skin biophysical characteristics in former-smokers - A study in postmenopausal women
}

\author{
Dorota Sagan', Jan Stepniak1, Adam Gesing1', Andrzej Lewinski',3, \\ Malgorzata Karbownik-Lewinska, ${ }^{1,3}$ \\ ${ }^{1}$ Department of Oncological Endocrinology, Medical University of Lodz, Lodz, Poland \\ 2 Department of Endocrinology and Metabolic Diseases, Medical University of Lodz, Lodz, Poland \\ 3 Polish Mother's Memorial Hospital - Research Institute, Lodz, Poland
}

Sagan D, Stepniak J, Gesing A, Lewinski A, Karbownik-Lewinska M. Melatonin reverses the enhanced oxidative damage to membrane lipids and improves skin biophysical characteristics in former-smokers - A study in postmenopausal women. Ann Agric Environ Med. 2017; 24(4): 659-666. doi: 10.5604/12321966.1235174

\begin{abstract}
Introduction and objective. Protective antioxidative effects of melatonin have been repeatedly documented in experimental and clinical studies. One of the most spectacular exogenous prooxidative agents is cigarette smoking. The aim of the study was to evaluate the level of oxidative damage to membrane lipids (lipid peroxidation; LPO) in blood serum, and in epidermis exfoliated during microdermabrasion collected from former-smokers who were treated with melatonin.

Materials and Method. The study was performed in postmenopausal women. Ninety (90) female volunteers, aged 46-67 years, were enrolled. Two major groups, i.e. never-smokers $(n=44)$ and former-smokers $(n=46)$, were divided into: Control, melatonin topical skin application, Restructurer (containing antioxidants) topical skin application, and melatonin oral treatment. Microdermabrasion was performed at point ' 0 ', after 2 weeks, and after 4 weeks of treatment. The following parameters were measured: LPO in blood serum, LPO in epidermis exfoliated during microdermabrasion, and skin biophysical characteristics, such as sebum, moisture, elasticity, and pigmentation. Malondialdehyde+4-hydroxyalkenals level (LPO index) was measured spectrophotometrically.

Results. Melatonin oral treatment significantly reversed the increased serum LPO level in former-smokers already after 2 weeks of treatment. In a univariate regression model, LPO blood level constituted the only independent factor negatively associated with melatonin oral treatment. After 4 weeks of treatment, melatonin given orally increased skin sebum, moisture and elasticity levels, and melatonin applied topically increased sebum level.

Conclusion. Exogenous melatonin reverses the enhanced oxidative damage to membrane lipids and improves skin biophysical characteristics in former-smokers.
\end{abstract}

\section{Key words}

smoking, former-smokers, never-smokers, postmenopausal women, oxidative stress, lipid peroxidation, skin biophysical characteristics, sebum, moisture, elasticity

\section{INTRODUCTION}

Biological effects of melatonin, as an antioxidant and free radical scavenger, have been repeatedly documented in numerous experimental and clinical studies and reviewed by experts in this area $[1,2]$. Melatonin has been found to protect against practically all pathological processes studied to-date [1-4]. Melatonin has been documented to prevent oxidative damage in different skin components $[5,6]$, which suggests that the human skin is a target for the protective actions of melatonin. It is also worth stressing that the human skin is a site of melatonin synthesis and metabolism, suggesting an important role for a local melatoninergic system in protection against oxidative damage to macromolecules [7]. Therefore, endogenous intracutaneous melatonin production and

Address for correspondence: Prof. Malgorzata Karbownik-Lewinska, Department of Oncological Endocrinology, Medical University of Lodz, Zeligowski 7/9, 90-752 Lodz, Poland.

Tel./Fax: 48426393121 (22)

E-mail: MKarbownik@hotmail.com

Received: 21 January 2016; accepted: 22 March 2016; first published on February 2017 topically-applied exogenous melatonin would be expected to represent one of the most potent anti-oxidative defence systems against oxidative damage to the skin components [7].

Oxidative stress is a process associated with the excess of reactive oxygen species (ROS), free radicals included, which damage all biological macromolecules, such as DNA, lipids and proteins. The increased oxidative damage to macromolecules may result from both exogenous agents or endogenous disturbances, both documented in clinical and experimental studies [8-15].

Cigarette smoking, being a risk factor for several diseases [16-18], is documented to be a well-known prooxidative agent $[19,20]$. It has been observed recently that past smoking is independently associated with increased damage to membrane lipids, regardless of the use of hormone replacement therapy (HRT) in postmenopausal women [21]. Different indices of oxidative damage to macromolecules are measured in the tissues and body fluids. Lipid peroxidation (LPO) results from oxidative damage to membrane lipids and is frequently evaluated in experimental and clinical studies [3, 4, 8-15, 22]. 
In studies in humans, LPO is usually measured in blood serum/plasma [9-12, 15, 23-25]. It is worth considering evaluating such parameters in components, which are routinely removed during different medical or cosmetic procedures. The authors have recently documented that LPO can be also measured in epidermis exfoliated from facial skin during microdermabrasion [22]. Microdermabrasion is a simple and non-invasive cosmetic technique in the treatment of several skin problems, frequently used in beauty parlours. In microdermabrasion, a mechanical medium is used for exfoliation to gently remove the outermost layer of dead skin cells from the epidermis [26].

It has also been documented that topical application of certain antioxidants enhances the clinical and histological changes seen following microdermabrasion alone [27]. Thus, it is worth checking if any other antioxidants are able to cause similar effects. Restructurer is a mixture of, among others, antioxidants, and is frequently used after microdermabrasion to improve its cosmetic effects. In the presented study, Restructurer was used to compare its effects with those of melatonin.

\section{OBJECTIVE}

The aim of the study was to evaluate the level of oxidative damage to membrane lipids in blood serum, and in epidermis exfoliated during microdermabrasion collected from neversmokers and from former-smokers treated with melatonin either orally or applied topically on the skin. For comparison, Restructurer was also used topically on the skin. The study was performed in postmenopausal women.

\section{MATERIALS AND METHOD}

The procedures used in the study were approved by the Ethical Committee of the Medical University of Lodz (Approval No. $\mathrm{RNN} / 205 / 09 / \mathrm{KE}$ ), and fully informed, written consent was obtained from the participants.

The following chemicals were used: Melatonin [SigmaAldrich (St. Louis, MO, USA] - for skin topical application; Restructurer (Aqua Csp 100\%; Ascorbic Acid 3\%, antioxidant; Methylsilanol Mannuonate 0.5\%, firming agent; Zinc Gluconate $0.173 \%$, Healing agent; Thioctic Acid $0.01 \%$, antioxidant; Manganese gluconate $0.01 \%$, regenerator; LABORATORIO INNOAESTHETICS, Pont Reixat 3, 08960 - Sant Just Desvern, Barcelona, Spain) - for topical skin application; Melatonin (5 mg tabletes, LEK-AM, Poland) for oral treatment.

A questionnaire containing questions related to major risk factors for civilization-related diseases (21) was polled in a group of female participants who were clients of a beauty parlour. Exclusion criteria constituted: cigarette smoking in the last 12 months, alcohol consumption, exposure to ionizing radiation or to any other potential prooxidative agent, and any acute or chronic disease.

As described above [21], ninety (90) adult female volunteers, aged 46-67 years, were finally enrolled in the study. Two major groups were considered, i.e. never-smokers $(n=44)$ and former-smokers $(n=46)$, which were additionally subgrouped into HRT users (HRT+) and HRT non-users (HRT). As statistical analysis performed at point ' 0 ' (published previously) [21] revealed no significant association with HRT treatment, the sub-groups of HRT users and HRT nonusers are not considered in the present study. The studied main groups were well matched at baseline (point ' 0 ') in terms of age and BMI (no statistically significant differences between never-smokers and former-smokers were found when evaluated by a Student's unpaired $t$ test) [21].

Both never-smokers and former-smokers were then divided to four subgroups according to the following treatment: Control (without any treatment), melatonin topical skin application, Restructurer topical skin application, and melatonin oral treatment (Tab. 1). Melatonin (0.5 mM; final volume $5 \mathrm{~mL}$ ) or Restructurer (final volume $5 \mathrm{~mL}$ ) were applied topically on the skin just after microdermabrasion. The concentration of $0.5 \mathrm{mM}$ of melatonin was chosen on the basis of the lowest concentration of one of the antioxidants in Restructurer (i.e. Thioctic Acid 0.01\%). Melatonin was also applied orally in a dose of $2.5 \mathrm{mg}$ every day, approx. 1 hour before bedtime.

Table 1. Treatment scheme in particular sub-groups of never-smokers and former-smokers

\begin{tabular}{lcc}
\hline & $\begin{array}{c}\text { never-smokers } \\
\mathrm{n}=44\end{array}$ & $\begin{array}{c}\text { former-smokers } \\
\mathrm{n}=46\end{array}$ \\
\hline $\begin{array}{l}\text { Control } \\
\text { No treatment }\end{array}$ & $\mathrm{n}=19$ & $\mathrm{n}=8$ \\
\hline $\begin{array}{l}\text { Melatonin } \\
\text { Topical skin application }\end{array}$ & $\mathrm{n}=11$ & $\mathrm{n}=10$ \\
\hline $\begin{array}{l}\text { Restructurer } \\
\text { Topical skin application }\end{array}$ & $\mathrm{n}=9$ & $\mathrm{n}=14$ \\
\hline $\begin{array}{l}\text { Melatonin } \\
\text { Oral treatment }\end{array}$ & $\mathrm{n}=5$ & $\mathrm{n}=14$ \\
\hline $\mathrm{n}$ - number of patients & &
\end{tabular}

Microdermabrasion was performed at three time points, i.e. at point ' 0 ' after 2 weeks and after 4 weeks of treatment. At the same time points, the following parameters were measured: LPO in blood serum, LPO in epidermis exfoliated during microdermabrasion, and four skin biophysical characteristics, such as sebum, moisture, elasticity, and pigmentation.

Microdermabrasion was performed by the use of a professional electroporation instrument - Meso In Derma (Rubika, Poland). Skin biophysical characteristics were measured with the use of Skin Analyzer [Bs-888pro(SMP)-V2] (Kowa SMEP; KOWA), and expressed as a percentage (i.e. 0-99\%).

Body mass and body height were measured to calculate $\mathrm{BMI}$, and waist circumference and hip circumference were measured to calculate waist/hip ratio (WHR). As described before, BMI did not differ between never-smokers and former-smokers at point '0' [21].

Blood samples were collected after an overnight fast. After collection, blood $(1 \mathrm{ml})$ was centrifuged $(3,000 \mathrm{x} \mathrm{g}, 10 \mathrm{~min}$, $4^{\circ} \mathrm{C}$ ) in order to obtain serum, and stored at $-80^{\circ} \mathrm{C}$ until assay. The outermost layer of dead skin cells of the epidermis was exfoliated from facial skin during microdermabrasion, and stored at $-80^{\circ} \mathrm{C}$ until assays.

LPO assay in blood serum. The concentrations of malondialdehyde + 4-hydroxyalkenals (MDA+4-HDA), as the index of LPO, were measured in blood serum using an LPO kit purchased from Enzo Life Science (Farmingdale, NY). The 
$\operatorname{serum}(200 \mathrm{ml})$ was mixed with $650 \mathrm{ml}$ of a methanol:acetonitrile $(1: 3, v / v)$ solution, containing a chromogenic reagent, $\mathrm{N}$-methyl-2-phenylindole, and vortexed. After adding 150 $\mathrm{ml}$ of methanesulfonic acid (15.4 M), incubation was carried out at $45^{\circ} \mathrm{C}$ for $40 \mathrm{~min}$. The reaction between $\mathrm{MDA}+4-\mathrm{HDA}$ and N-methyl-2-phenylindole yielded a chromophore which was spectrophotometrically measured at the absorbance of $586 \mathrm{~nm}$, using a solution of 4-hydroxynonenal $(10 \mathrm{mM})$ as the standard. The level of LPO was expressed as the amount of MDA+4-HDA (nmol) per $1 \mathrm{ml}$ of serum.

LPO assay in the epidermis. The concentrations of malondialdehyde + 4-hydroxyalkenals (MDA+4-HDA), as the index of LPO, were measured in epidermis exfoliated during microdermabrasion, using an LPO kit, purchased from Enzo Life Science (Farmingdale, NY). The epidermis $(1 \mathrm{mg})$ was homogenized in ice-cold $50 \mathrm{mM}$ Tris buffer $(\mathrm{pH}$ $7.4,10 \%, \mathrm{w} / \mathrm{v})$. After centrifugation at $10,000 \mathrm{~g}$ for $10 \mathrm{~min}$ at $4^{\circ} \mathrm{C}$, the supernatants $(200 \mathrm{ml})$ were mixed with $650 \mathrm{ml}$ of a methanol:acetonitrile $(1: 3, \mathrm{v} / \mathrm{v})$ solution, containing a chromogenic reagent, $\mathrm{N}$-methyl-2-phenylindole, and vortexed. After adding $150 \mathrm{ml}$ of methanesulfonic acid (15.4 $\mathrm{M})$, incubation was carried out at $45^{\circ} \mathrm{C}$ for $40 \mathrm{~min}$. The reaction between MDA+4-HDA and N-methyl-2-phenylindole yielded a chromophore which was spectrophotometrically measured at the absorbance of $586 \mathrm{~nm}$, using a solution of 4-hydroxynonenal $(10 \mathrm{mM})$ as the standard. The level of LPO was expressed as the amount of MDA+4-HDA (nmol) per $1 \mathrm{mg}$ of epidermis.
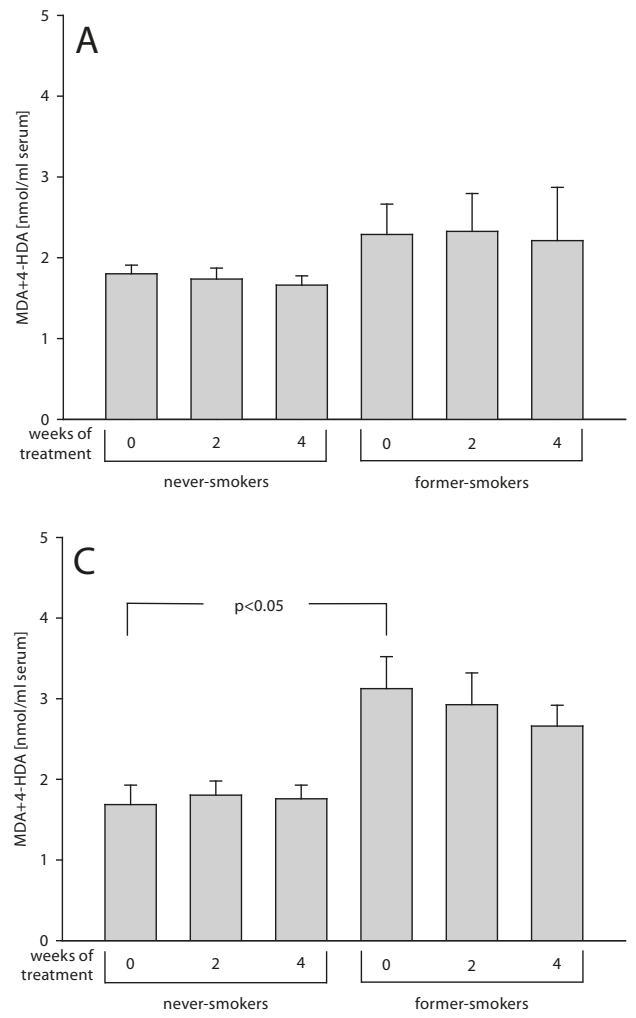

Statistical analysis. The data were statistically analysed using an unpaired or a paired Student's $t$ test for two independent variables or two dependent (paired) variables, respectively. The results are presented as means \pm SEM. Univariate logistic regression analysis was used to determine which continuous variable might have been associated with melatonin oral treatment. Statistical significance was determined at the level of $\mathrm{p}<0.05$.

\section{RESULTS}

The level of LPO products in blood serum was evaluated in never-smokers and in former-smokers after two weeks and after four weeks of one of three kinds of treatment (Fig. 1). At point ' 0 ', serum LPO level was higher in former-smokers than in never-smokers, with statistical significance reached only in one of the sub-groups (Fig. 1C), but this difference was clearly observed when all former-smokers were compared with all never-smokers [21]. In the group of never-smokers, serum LPO level had not changed in response to any treatment (Fig. 1A, B, C, D). Melatonin oral treatment significantly reversed the increased serum LPO in former-smokers already after two weeks of treatment (Fig. 1D). Topical application of either melatonin or Restructurer did reveal protective effects against oxidative damage to membrane lipids in formersmokers (Fig. 1B, C).

Concerning the level of LPO products measured in the epidermis, it did not differ significantly, neither between
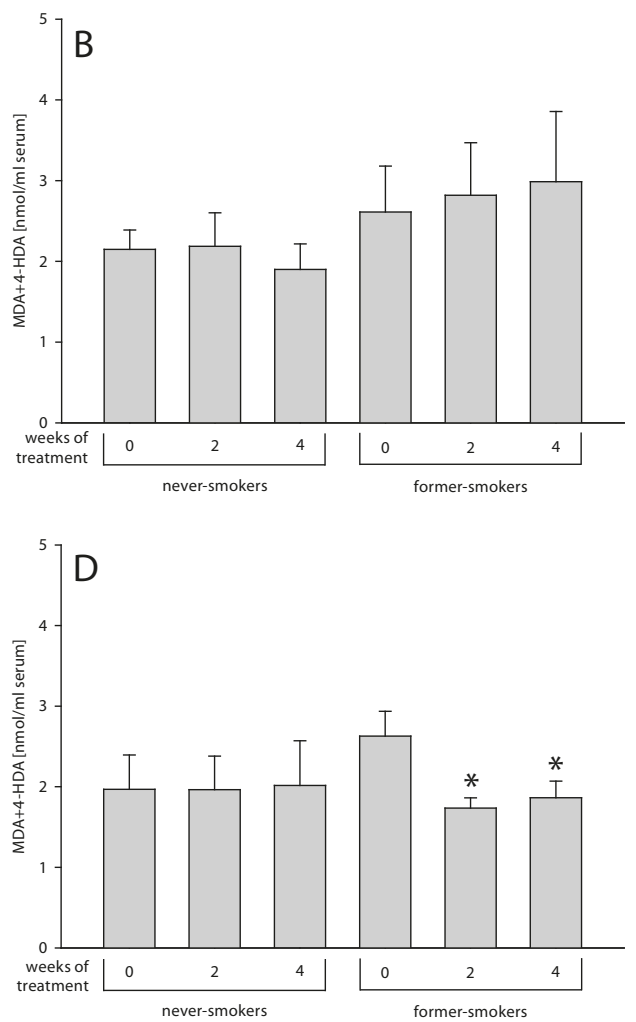

Figure 1. Mean ( \pm SEM) values of blood lipid peroxidation $(L P O)$ in never-smokers $(n=19)$ and in former-smokers $(n=8)$ without any treatment (Control, $A)$; in never-smokers $(n=11)$ and in former-smokers $(n=10)$ with topical melatonin application (B); in never-smokers $(n=9)$ and in former-smokers $(n=14)$ with topical Restructurer application $(C)$; and in never-smokers $(n=5)$ and in former-smokers $(n=14)$ with oral melatonin treatment (D) at point ' 0 ', after 2 weeks (' 2 ') and after 4 weeks (' 4 ') of treatment.

Statistical evaluation was performed by a paired Student's t-test within one sub-group or by an unpaired Student's t-test between sub-groups. The level of LPO is expressed as the amount of MDA+4-HDA (nmol) per $1 \mathrm{ml}$ of serum.

${ }^{*} p<0.05$ vs. point ' 0 ' of former-smokers; other statistical significance is marked in the Figure if applicable. 

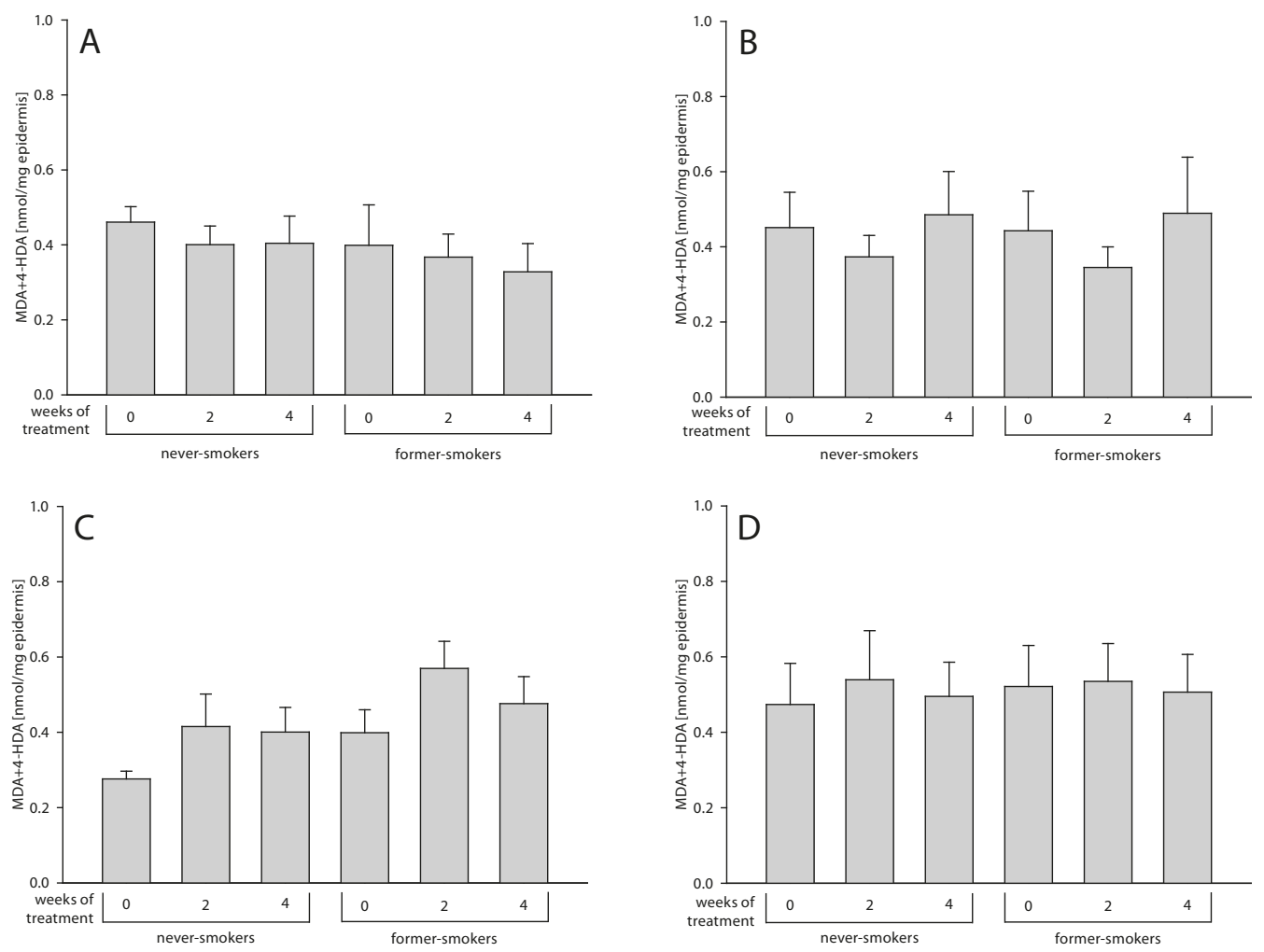

Figure 2. Mean ( \pm SEM) values of lipid peroxidation (LPO) in epidermis in never-smokers $(n=19)$ and in former-smokers $(n=8)$ without any treatment (Control, A); in never-smokers $(n=11)$ and in former-smokers $(n=10)$ with topical melatonin application $(B)$; in never-smokers $(n=9)$ and in former-smokers ( $n=14)$ with topical Restructurer application (C); and in never-smokers $(n=5)$ and in former-smokers ( $n=14)$ with oral melatonin treatment (D) at point ' 0 ', after 2 weeks (' 2 ') and after 4 weeks (' 4 ') of treatment.

Statistical evaluation was performed by a paired Student's t-test within one subgroup or by an unpaired Student's t-test between subgroups. The level of LPO is expressed as the amount of MDA+4-HDA (nmol) per $1 \mathrm{mg}$ of epidermis. No statistical significance was found.
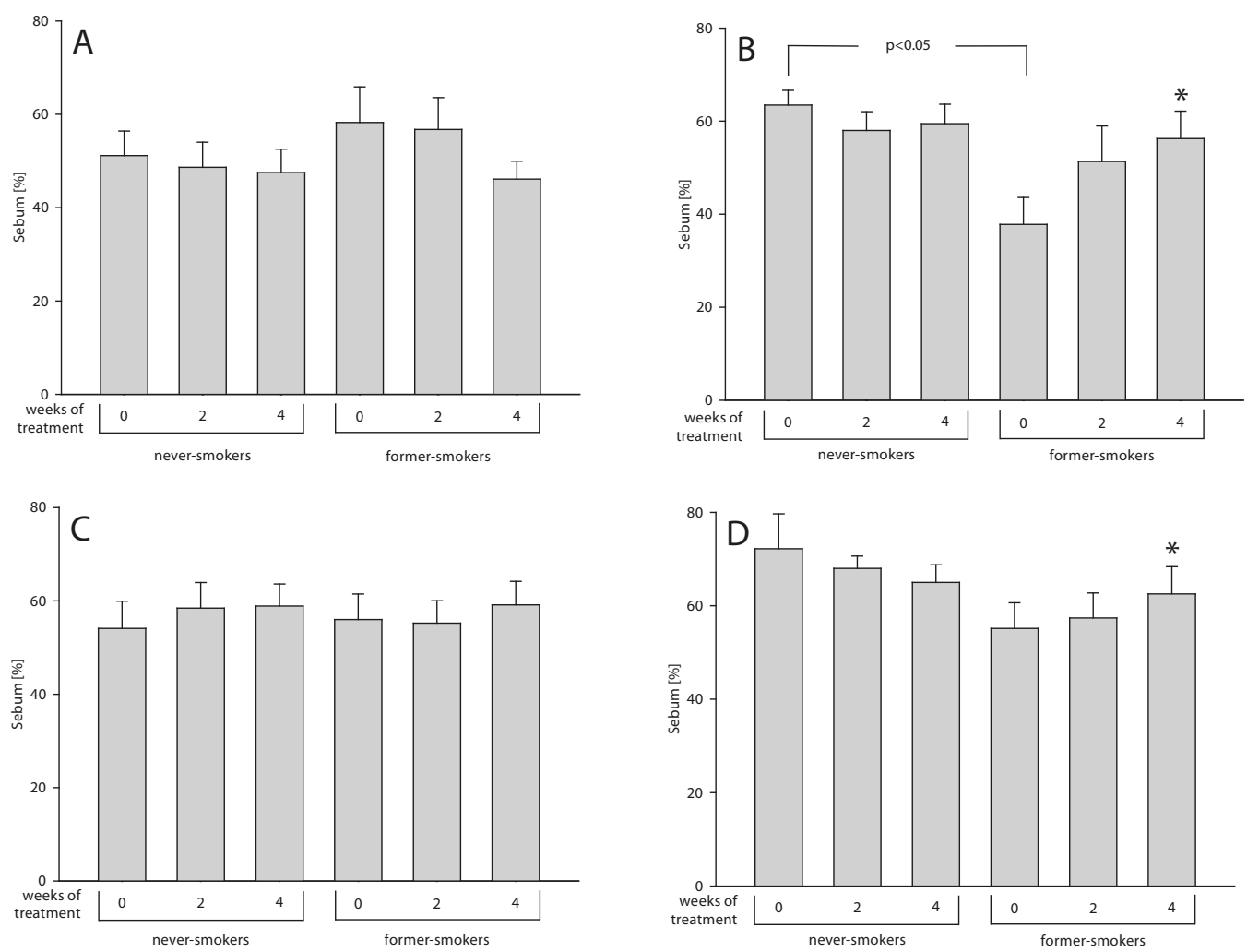

Figure 3. Mean ( \pm SEM) values of sebum [\%] in never-smokers $(n=19)$ and in former-smokers $(n=8)$ without any treatment $($ Control, $A)$; in never-smokers $(n=11)$ and in former-smokers $(n=10)$ with topical melatonin application $(B)$; in never-smokers $(n=9)$ and in formersmokers ( $n=14)$ with topical Restructurer application $(C)$; and in never-smokers $(n=5)$ and in former-smokers $(n=14)$ with oral melatonin treatment (D) at point " 0 ", after 2 weeks (' 2 ') and after 4 weeks (' 4 ') of treatment.

Statistical evaluation was performed by a paired Student's t-test within one sub-group or by an unpaired Student's t-test between sub-groups. The level of sebum is expressed as a percentage (i.e. 0-99\%).

${ }^{*} p<0.05$ vs. point ' 0 ' of former-smokers; other statistical significance is marked in the Figure if applicable. 

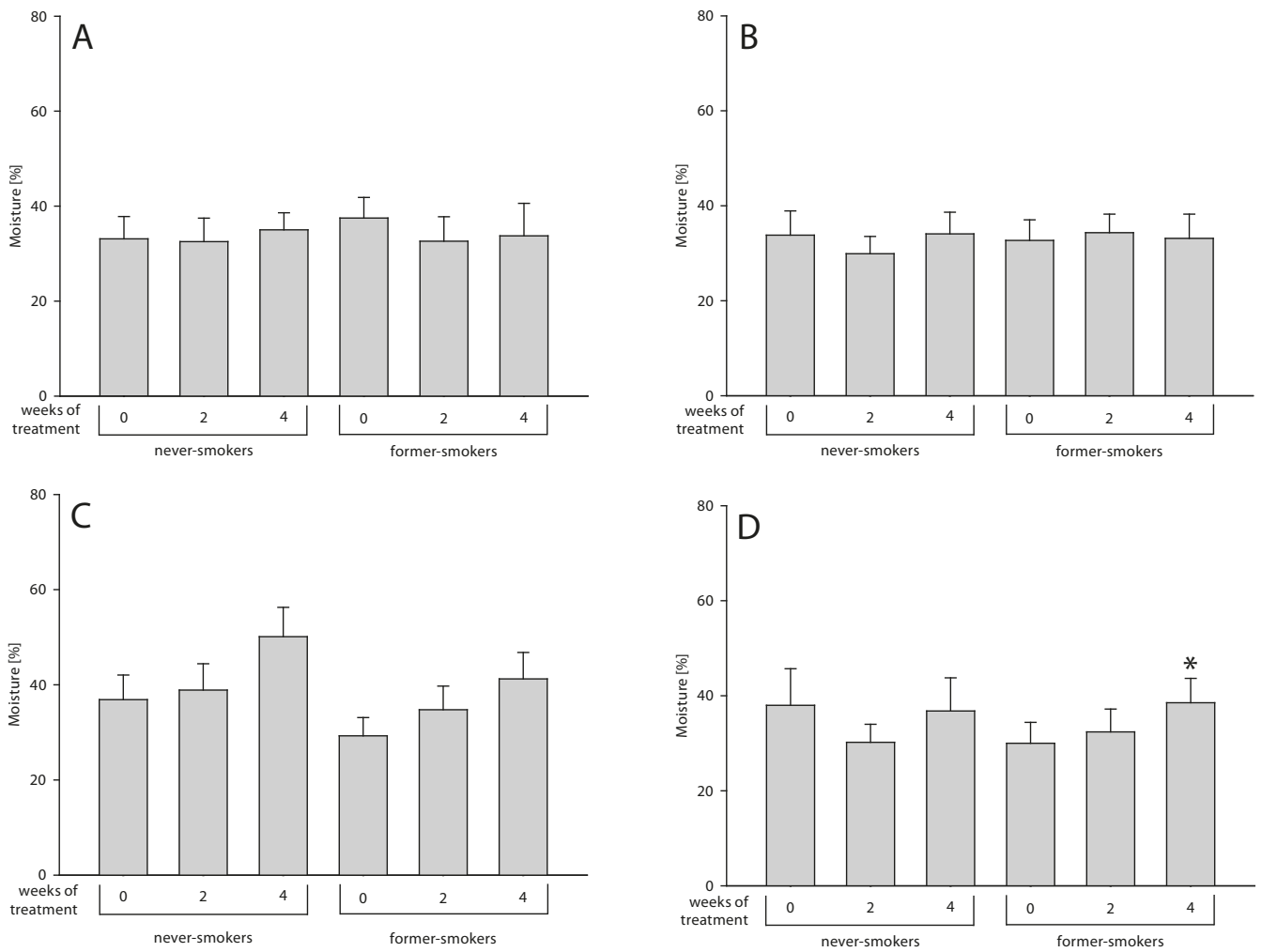

Figure 4. Mean $( \pm$ SEM) values of moisture $[\%]$ in never-smokers $(n=19)$ and in former-smokers $(n=8)$ without any treatment $($ Control, A); in never-smokers $(n=11)$ and in former-smokers $(n=10)$ with topical melatonin application $(B)$; in never-smokers $(n=9)$ and in formersmokers ( $n=14)$ with topical Restructurer application $(C)$; and in never-smokers $(n=5)$ and in former-smokers $(n=14)$ with oral melatonin treatment (D) at point ' 0 ', after 2 weeks (' 2 ') and after 4 weeks (' 4 ') of treatment.

Statistical evaluation was performed by a paired Student's t-test within one sub-group or by an unpaired Student's t-test between sub-groups. The level of moisture was expressed as a percentage (i.e. 0-99\%).

${ }^{*} \mathrm{p}<0.05$ vs. point ' 0 ' of former-smokers; other statistical -significance is marked in the Figure if applicable.
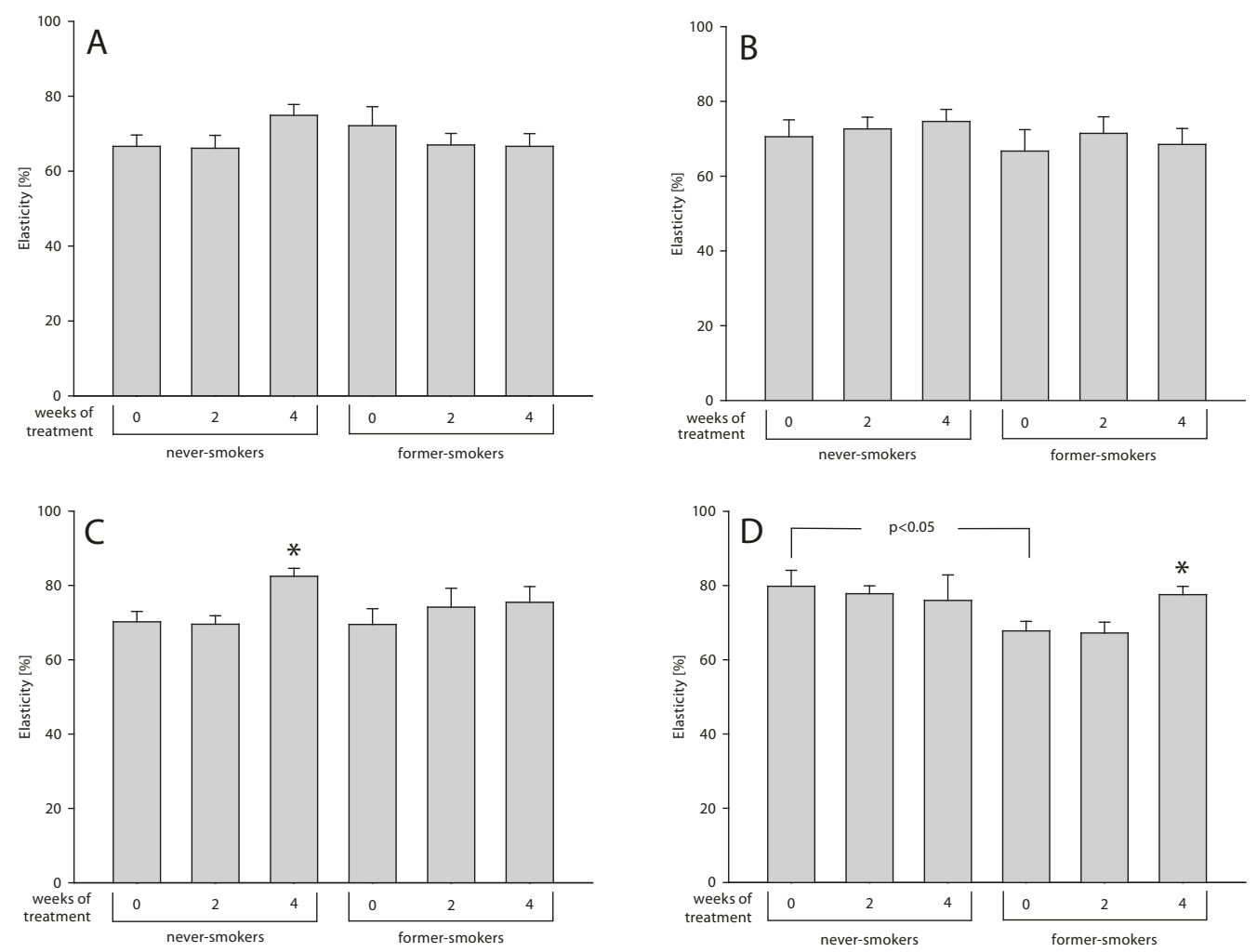

Figure 5. Mean $( \pm$ SEM) values of elasticity [\%] in never-smokers $(n=19)$ and in former-smokers $(n=8)$ without any treatment $($ Control, A); in never-smokers ( $n=11)$ and in former-smokers $(n=10)$ with topical melatonin application $(B)$; in never-smokers $(n=9)$ and in formersmokers $(n=14)$ with topical Restructurer application $(C)$; and in never-smokers $(n=5)$ and in former-smokers $(n=14)$ with oral melatonin treatment (D) at point ' 0 ', after 2 weeks (' 2 ') and after 4 weeks (' 4 ') of treatment.

Statistical evaluation was performed by a paired Student's t-test within one sub-group or by an unpaired Student's t-test between sub-groups. The level of elasticity was expressed as a percentage (i.e. 0-99\%).

${ }^{*} p<0.05$ vs. point ' 0 ' of former-smokers; other statistical significance is marked in the Figure if applicable. 

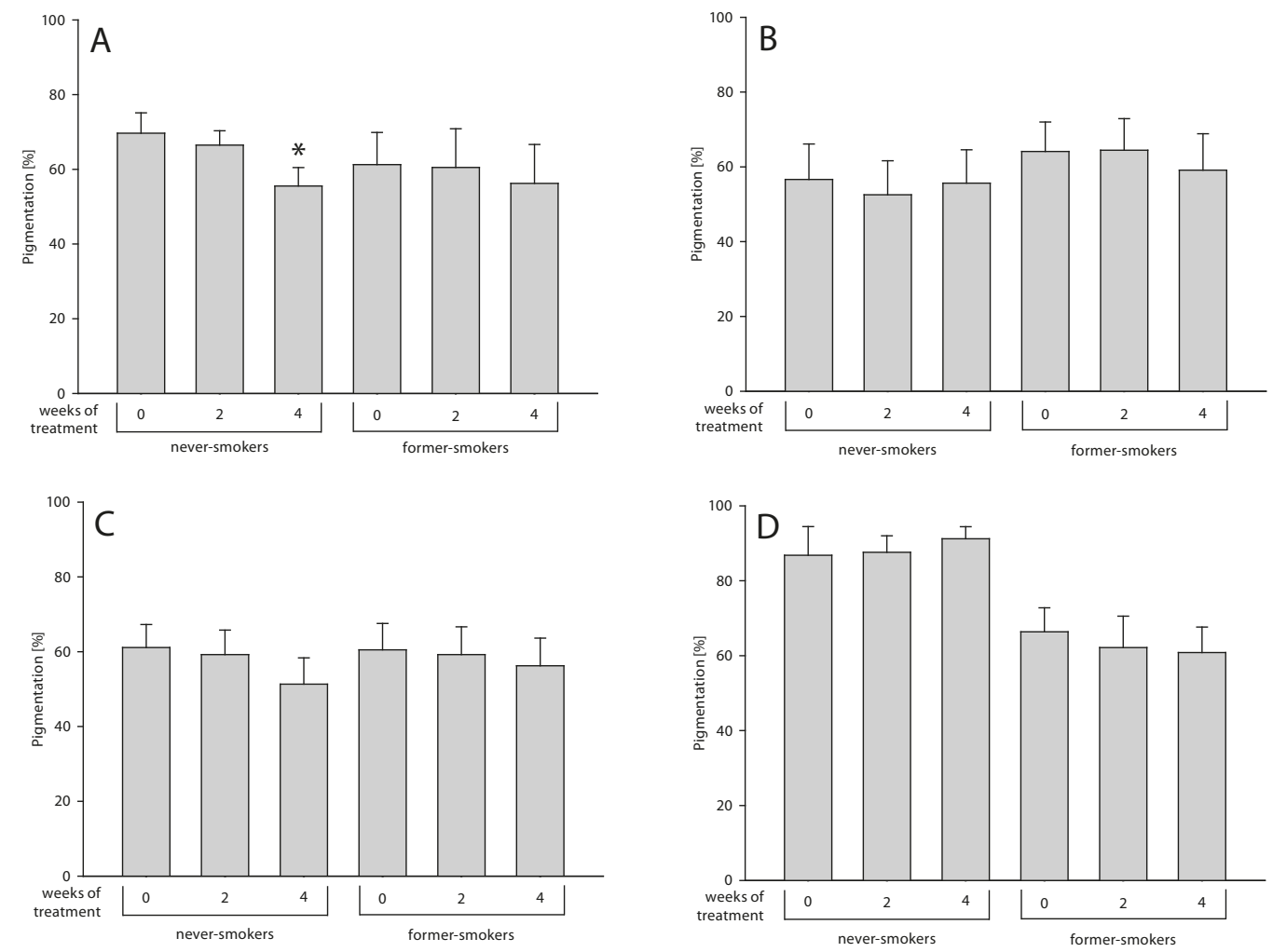

Figure 6. Mean $( \pm$ SEM) values of pigmentation [\%] in never-smokers $(n=19)$ and in former-smokers $(n=8)$ without any treatment $(C o n t r o l$, $A)$; in neversmokers $(n=11)$ and in former-smokers $(n=10)$ with topical melatonin application (B); in never-smokers $(n=9)$ and in former-smokers $(n=14)$ with topical Restructurer application (C); and in never-smokers $(n=5)$ and in former-smokers $(n=14)$ with oral melatonin treatment (D) at point ' 0 ', after 2 weeks (' 2 ') and after 4 weeks ('4') of treatment.

Statistical evaluation was performed by a paired Student's t-test within one sub-group or by an unpaired Student's t-test between sub-groups. The level of pigmentation was expressed as a percentage (i.e. 0-99\%). No statistical significance was found.

never-smokers and former-smokers nor within sub-groups in response to any treatment (Fig. 2A, B, C, D).

Biophysical characteristics, such as sebum, moisture, elasticity and pigmentation, were evaluated in never-smokers and in former-smokers after two weeks and after four weeks of one of the three kinds of treatment (Fig. 3-6).

Concerning sebum (Fig. 3), only in one of sub-groups (Fig. 3B) its degree was significantly lower in former-smokers than in never-smokers. When the results were analyzed in former-smokers, melatonin either applied topically on the skin (Fig. 3B) or given orally (Fig. 3D), increased the degree of sebum after four weeks of treatment. The treatment with Restructurer did not change the sebum level (Fig. 3C).

The level of moisture did not differ between formersmokers and never-smokers (Fig. 4). Melatonin applied orally significantly increased the level of moisture in formersmokers after four weeks of treatment (Fig. 4D).

The level of elasticity did not differ between formersmokers and never-smokers (Fig. 5). Melatonin applied orally significantly increased the level of moisture in formersmokers after four weeks of treatment (Fig. 5D). Additionally, Restructurer applied in topical treatment significantly increased the level of elasticity in never-smokers after four weeks of treatment (Fig. 5C).

The level of pigmentation did not differ between formersmokers and never-smokers (Fig. 6). Melatonin applied topically on the skin or orally did not change the level of pigmentation (Fig. 6B,D). Microdermabrasion alone resulted
Table 2. Univariate logistic regression analysis of the melatonin treatment variables (determinants), performed in all former smokers $(n=46)$ after 2 weeks of treatment

\begin{tabular}{lccc}
\hline \multirow{2}{*}{ Variable } & \multicolumn{3}{c}{ Univariate regression } \\
\cline { 2 - 4 } & OR & $95 \% \mathrm{Cl}$ & $\mathrm{p}$ \\
\hline Age [years] & 0.920 & $0.78-1.07$ & 0.291 \\
\hline Body mass [kg] & 1.004 & $0.95-1.05$ & 0.865 \\
\hline Height [m] & 0.140 & $0.000002-11175.89$ & 0.725 \\
\hline BMI [kg/m2] & 1.027 & $0.88-1.19$ & 0.720 \\
\hline Waist circumference $[\mathrm{cm}]$ & - & - & - \\
\hline Hip circumference $[\mathrm{cm}]$ & 1.007 & $0.94-1.07$ & 0.834 \\
\hline WHR & 0.118 & $0.0000007-19103.64$ & 0.719 \\
\hline LPO blood level & 0.307 & $0.08-1.06$ & 0.055 \\
\hline MDA + 4-HDA $(\mathrm{nmol} / \mathrm{ml})]$ & 1.475 & $0.40-5.41$ & 0.545 \\
\hline LPO epidermis level & 1.003 & $0.97-1.03$ & 0.827 \\
\hline MDA + 4-HDA $(\mathrm{nmol} / \mathrm{ml})]$ & 0.987 & $0.94-1.02$ & 0.525 \\
\hline Sebum & 0.973 & $0.92-1.02$ & 0.271 \\
\hline Moisture & 0.999 & $0.97-1.02$ & 0.976 \\
\hline Elasticity & $-b 00$ & & \\
\hline Pigmentation & & & \\
\hline
\end{tabular}

OR - odds ratio; Cl - confidence interval; BMI - body mass index; WHR - waist/hip ratio; LPO lipid peroxidation; MDA + 4-HAD - malondialdehyde + 4-hydroxyalkenals.

Level of statistical significance is given in italic, with statistical significance accepted at the level of $\mathrm{p}<0.05$.

in a decreased level of pigmentation in the sub-group of never smokers (Fig. 6A). 
For the group of former-smokers, variables such as age, body mass, height, BMI, waist circumference, hip circumference and waist-hip ratio (WHR), blood LPO level, LPO level in epidermis, and biophysical characteristics, such as sebum, moisture, elasticity and pigmentation, were submitted to a univariate regression model. The purpose of the model was to determine which of those continuous variables might have been independently associated with melatonin oral treatment. The blood level of LPO constitute the only independent factor negatively associated with melatonin oral treatment when evaluated after two weeks of treatment (Tab. 2). When evaluated after four weeks of treatment, the above association lost its statistical significance (data not shown).

\section{DISCUSSION}

In the present study, in the same population as before, i.e. in former-smokers and in never-smokers [21], the response to melatonin treatment was evaluated. Restructurer, being a mixture frequently used in beauty parlours to improve the effects of microdermabrasion, was used topically on the skin to compare its effect with the effect of topical melatonin treatment.

The increased LPO in former-smokers, documented in a study published previously [21], has only been partially confirmed in the presented study, which is the result of the much smaller samples of sub-groups analyzed in this study.

Effects of melatonin treatment in never-smokers. As expected, melatonin given either orally or applied directly on the skin, did not change the level of measured parameters in blood serum or in the skin. These findings are in agreement with the assumption that melatonin does not affect processes at the physiological level. Such an assumption has been repeatedly confirmed in experimental and clinical studies in which melatonin used separately did not change the level of oxidative damage to macromolecules but, at the same time, it revealed protective effects against prooxidative agents [3, $4,8,28-32]$.

Effects of metalonin in former-smokers. Melatonin given orally completely reversed the increased blood LPO already after two weeks of treatment. Additionally, this favourable effect of melatonin was confirmed in regression analysis in which LPO constituted the only independent determinant (variable) negatively associated with melatonin treatment. This finding suggests that lower LPO levels in formersmokers are directly associated with the treatment with exogenous melatonin. Similarly, in earlier studies, such short time as days or weeks of melatonin treatment, was sufficient to reverse oxidative damage to macromolecules in animals $[28,29]$ and in humans $[31,33]$.

Concerning the protective influence of melatonin on deleterious effects caused by smoking, there are some studies published which concern this protective influence, but they relate to current smoking $[32,34]$. The presented study is the first to document protective effects of melatonin against oxidative damage to macromolecules in formersmokers.

LPO measured in epidermis. The level in former-smokers did not differ from that found in never-smokers. However, the results of the current study are not discrepant, as LPO products present in the blood come from practically all tissues and organs, which - at least to a certain extent - are pathologically changed in former-smokers, whereas those present in the dead cells of the epidermis are predominantly produced only locally. The level of LPO products in epidermis may be changed by other prooxidative agents, such as overweight and obesity, which has been observed previously by the authors in female adults [22]. Thus, overweight and obesity are probably stronger pro-oxidative agents than past smoking, at least concerning their influence on skin components.

In the light of the present results, it could be suggested that a similar study evaluating LPO in the epidermis would be worth performing in current-smokers.

Concerning the influence of melatonin treatment, the antioxidant given orally or applied topically on the skin did not reveal any effect on LPO level in epidermis, as was expected. Similarly, Restructurer did not affect LPO in the epidermis.

Biophysical characteristics of facials skin. These did not differ between former-smokers and never-smokers, with only sebum - to a certain extant - being higher in neversmokers. The reason of this apparent lack of differences could be related to the fact that former-smokers were studied instead of current-smokers, and the period of twelve months of no smoking could be long enough to reverse facial skin changes related to cigarette exposure. In turn, biophysical characteristics of facials skin were positively affected by melatonin in former-smokers; namely, sebum content, moisture and elasticity were changed favourably in response to melatonin oral treatment. Concerning the first characteristic, this was improved additionally after topical treatment with melatonin.

Thus, the results of the presented study suggest that melatonin should be considered as an agent to be used as a topical antioxidant to enhance the effects of facial microdermabrasion. This study is the first to show the favourable effects of melatonin on skin biophysical characteristics.

The main limitation of this study is that it was performed in a specific group of patients, i.e. in postmenopausal women, in whom oxidative processes may be affected by two main factors: aging and physiological hormonal changes, which affect the quality of the skin, which is usually lower in postmenopausal women. A similar study should be performed in other specific groups of subjects or just in the general population, the results of which will possibly confirm the presented findings. Other limitations are that no passive smoking was evaluated in the study, especially as a relationship has been documented between sensitivity to passive and to active smoking [35], and the small sample size, especially in the sub-groups, also constituted a limitation.

\section{CONCLUSIONS}

Exogenous melatonin reverses enhanced oxidative damage to membrane lipids and improves skin biophysical characteristics in former-smokers. Taking into account the safety of melatonin [36], this molecule should be considered in the prevention of consequences of smoking, not only in postmenopausal women, but also in the whole population. 
Melatonin should also be considered as a topical antioxidant to enhance the effects of facial microdermabrasion.

\section{Acknowledgements}

The research was supported by the Medical University of Lodz, Poland (No. 503/1-168-01/503-11-001). The results presented in this study constitute a part of the Ph.D. thesis of the first author.

\section{Conflict of interest}

The authors declare that they have no conflict of interests that could be perceived as prejudicing the impartiality of the presented study.

\section{REFERENCES}

1. Tan DX, Manchester LC, Esteban-Zubero E, Zhou Z, Reiter RJ. Melatonin as a Potent and Inducible Endogenous Antioxidant: Synthesis and Metabolism. Molecules 2015; 20(10): 18886-18906.

2. Manchester LC, Coto-Montes A, Boga JA, Andersen LP, Zhou Z, Galano

A, Vriend J, Tan DX, Reiter RJ. Melatonin: an ancient molecule that makes oxygen metabolically tolerable. J Pineal Res. 2015; 59(4): 403-419.

3. Karbownik M, Lewinski A, Reiter RJ. Anticarcinogenic actions of melatonin which involve antioxidative processes: comparison with other antioxidants. Int J Biochem Cell Biol. 2001; 33(8): 735-753.

4. García JJ, López-Pingarrón L, Almeida-Souza P, Tres A, Escudero P, García-Gil FA, Tan DX, Reiter RJ, Ramírez JM, Bernal-Pérez M. Protective effects of melatonin in reducing oxidative stress and in preserving the fluidity of biological membranes: a review. J Pineal Res. 2014; 56(3): 225-37.

5. Janjetovic Z, Nahmias ZP, Hanna S, Jarrett SG, Kim TK, Reiter RJ, Slominski AT. Melatonin and its metabolites ameliorate ultraviolet B-induced damage in human epidermal keratinocytes. J Pineal Res. 2014; 57(1): 90-102.

6. Kleszczyński K, Zwicker S, Tukaj S, Kasperkiewicz M, Zillikens D, Wolf R, Fischer TW. Melatonin compensates silencing of heat shock protein 70 and suppresses ultraviolet radiation-induced inflammation in human skin ex vivo and cultured keratinocytes. J Pineal Res. 2015; 58(1): 117-126.

7. Slominski AT, Kleszczyński K, Semak I, Janjetovic Z, Zmijewski MA, Kim TK, Slominski RM, Reiter RJ, Fischer TW. Local melatoninergic system as the protector of skin integrity. Int J Mol Sci. 2014; 15(10): 17705-17732.

8. Karbownik M, Lewinski A. Melatonin reduces Fenton reaction-induced lipid peroxidation in porcine thyroid tissue. J Cell Biochem. 2003; 90(4): 806-811.

9. Karbownik-Lewinska M, Gesing A, Zasada K, Jedrzejczyk M, Sobieszczanska-Jablonska A, Krawczyk J, et al. Relationship between lipid peroxidation or carcinoembryonic antigen and risk factors for non-communicable diseases in women at midlife and beyond. Neuro Endocrinol Lett. 2012; 33(5): 536-545.

10. Karbownik-Lewinska M, Kokoszko A, Lewandowski K, Shalet SM, Lewinski A. Growth hormone replacement reduces increased lipid peroxidation in growth hormone-deficient adults. Clin Endocrinol. 2008; 68: 957-964

11. Karbownik-Lewinska M, Szosland J, Kokoszko-Bilska A, Stępniak J, Zasada K, Gesing A, et al. Direct contribution of obesity to oxidative damage to macromolecules. Neuro Endocrinol Lett. 2012; 33(4): $453-461$.

12. Lewandowski KC, Stojanovic N, Press M, Tuck S, Lewiński A, Karbownik-Lewińska M. Raised concentrations of lipid peroxidation products (LPO) in pregnant women with impaired glucose tolerance. Ann Agric Environ Med. 2014; 21(2): 429-434.

13. Milczarek M, Stępniak J, Lewiński A, Karbownik-Lewińska M. Potassium iodide, but not potassium iodate, as a potential protective agent against oxidative damage to membrane lipids in porcine thyroid. Thyroid Res. 2013; 6(1): 10.

14. Stępniak J, Lewiński A, Karbownik-Lewińska M. Membrane lipids and nuclear DNA are differently susceptive to Fenton reaction substrates in porcine thyroid. Toxicol In Vitro. 2013; 27(1): 71-78.

15. Szychta P, Zadrozny M, Lewinski A, Karbownik-Lewinska M. Increased oxidative damage to membrane lipids following surgery for breast cancer. Neuro Endocrinol Lett. 2014; 35(7): 602-607.
16. Domej W, Oettl K, Renner W. Oxidative stress and free radicals in COPD--implications and relevance for treatment. Int J Chron Obstruct Pulmon Dis. 2014; 9: 1207-1224.

17. Goldkorn T, Filosto S, Chung S. Lung injury and lung cancer caused by cigarette smoke-induced oxidative stress: Molecular mechanisms and therapeutic opportunities involving the ceramide-generating machinery and epidermal growth factor receptor. Antioxid Redox Signal. 2014; 21(15): 2149-2174.

18. Siasos G, Tsigkou V, Kokkou E, Oikonomou E, Vavuranakis M, Vlachopoulos C, et al. Smoking and atherosclerosis: mechanisms of disease and new therapeutic approaches. Curr Med Chem. 2014; 21(34): 3936-3948.

19. Hakim IA, Harris R, Garland L, Cordova CA, Mikhael DM, Sherry Chow HH. Gender difference in systemic oxidative stress and antioxidant capacity in current and former heavy smokers. Cancer Epidemiol Biomarkers Prev. 2012; 21(12): 2193-2200.

20. Haswell LE, Papadopoulou E, Newland N, Shepperd CJ, Lowe FJ. A cross-sectional analysis of candidate biomarkers of biological effect in smokers, never-smokers and ex-smokers. Biomarkers. 2014; 19(5): 356-367.

21. Sagan D, Stepniak J, Gesing A, Lewinski A, Karbownik-Lewinska M. Higher lipid peroxidation in former-smokers vs.never-smokers - study in postmenopausal women. Neuro Endocrinol Lett. 2015; 36(6): 101-107.

22. Szokalska K, Stepniak J, Karbownik-Lewinska M. Lipid peroxidation evaluated in epidermis exfoliated during microdermabrasion is a reliable marker of oxidative stress related to obesity. J Eur Acad Dermatol Venereol. 2015 in print.

23. Kasperczyk S, Dobrakowski M, Kasperczyk A, Zalejska-Fiolka J, Pawlas N, Kapka-Skrzypczak L, Birkner E. Effect of treatment with $\mathrm{N}$-acetylcysteine on non-enzymatic antioxidant reserves and lipid peroxidation in workers exposed to lead. Ann Agric Environ Med. 2014; 21(2): 272-277.

24. Krzeszowiak J, Zawadzki M, Markiewicz-Górka I, Kawalec A, Pawlas $\mathrm{K}$. The influence of 9-day trekking in the Alps on the level of oxidative stress parameters and blood parameters in native lowlanders. Ann Agric Environ Med. 2014; 21(3): 585-589.

25. Łuczaj W, Moniuszko A, Rusak M, Zajkowska J, Pancewicz S, Skrzydlewska E. Peroxidative metabolism of arachidonic acid in the course of Lyme arthritis. Ann Agric Environ Med. 2015; 22(3): 433-437.

26. Kirkland EB, Hantash BM. Microdermabrasion: molecular mechanisms unraveled, part 1. J Drugs Dermatol. 2012; 11: 2-9.

27. Freedman BM. Topical antioxidant application enhances the effects of facial microdermabrasion. J Dermatolog Treat. 2009; 20(2): 82-87.

28. Karbownik M, Tan D, Manchester LC, Reiter RJ. Renal toxicity of the carcinogen delta-aminolevulinic acid: antioxidant effects of melatonin. Cancer Lett. 2000; 161(1): 1-7.

29. Karbownik M, Stasiak M, Zygmunt A, Zasada K, Lewiński A. Protective effects of melatonin and indole-3-propionic acid against lipid peroxidation, caused by potassium bromate in the rat kidney. Cell Biochem Funct. 2006; 24(6): 483-489.

30. Karbownik M, Reiter RJ. Melatonin protects against oxidative stress caused by delta-aminolevulinic acid: implications for cancer reduction. Cancer Invest. 2002; 20(2): 276-286.

31. Gitto E, Karbownik M, Reiter RJ, Tan DX, Cuzzocrea S, Chiurazzi P, Cordaro S, Corona G, Trimarchi G, Barberi I. Effects of melatonin treatment in septic newborns. Pediatr Res. 2001; 50(6): 756-60.

32. Shin IS, Shin NR, Park JW, Jeon CM, Hong JM, Kwon OK, Kim JS, Lee IC, Kim JC, Oh SR, Ahn KS. Melatonin attenuates neutrophil inflammation and mucus secretion in cigarette smoke-induced chronic obstructive pulmonary diseases via the suppression of ErkSp1 signaling. J Pineal Res. 2015; 58(1): 50-60.

33. Bejarano I, Monllor F, Marchena AM, Ortiz A, Lozano G, Jiménez MI, Gaspar P, García JF, Pariente JA, Rodríguez AB, Espino J. Exogenous melatonin supplementation prevents oxidative stress-evoked DNA damage in human spermatozoa. J Pineal Res. 2014; 57(3): 333-339.

34. Yang GH, Li YC, Wang ZQ, Liu B, Ye W, Ni L, Zeng R, Miao SY, Wang LF, Liu CW. Protective effect of melatonin on cigarette smoke-induced restenosis in rat carotid arteries after balloon injury. J Pineal Res. 2014; 57(4): 451-458.

35. Tarnoki AD, Littvay L, Lazar Z, Karlinger K, Molnar AA, Melicher D, Garami Z, Berczi V, Horvath I. Transmission of second-hand smoke sensitivity and smoking attTarnoki DLitude in a family. Ann Agric Environ Med. 2014; 21(4): 771-5.

36. Andersen LP, Gögenur I, Rosenberg J, Reiter RJ. The Safety of Melatonin in Humans. Clin Drug Investig 2015 in print. 